\title{
Bisoxazolomycin A: a new natural product from 'Streptomyces subflavus subsp. irumaensis' AM-3603
}

\author{
Wilaiwan Koomsiri ${ }^{1,5}$, Yuki Inahashi ${ }^{2,3,5}$, Tōru Kimura ${ }^{3}$, Kazuro Shiomi ${ }^{2,3}$, Yōko Takahashi ${ }^{2}$, Satoshi Ōmura ${ }^{2}$, \\ Arinthip Thamchaipenet ${ }^{1,4}$ and Takuji Nakashima ${ }^{2,3}$
}

Bisoxazolomycin (1), oxazolomycin A2 (2) and oxazolomycin A (3) were identified by physicochemical screening approach from a culture broth of 'Streptomyces subflavus subsp. irumaensis' AM-3603. Compound 2 is a hydrolyzed analog of 3 at the $\beta$-lactone ring, and 1 is a new dimeric analog of 2 . Compounds 1 and 2 exhibited less potent antibacterial activity and cytotoxicity than 3 , which might be due to lack of the $\beta$-lactone ring.

The Journal of Antibiotics (2017) 70, 1142-1145; doi:10.1038/ja.2017.113; published online 27 September 2017

\section{INTRODUCTION}

Our continuing search for new compounds from culture broths of microorganisms exploits innovative physicochemical (PC) screening, which is based on the physicochemical properties of compounds using LC-UV/vis diode array detection, LC/MS and color reaction. ${ }^{1}$ In the course of our PC screening for new compounds, the culture broth of 'Streptomyces subflavus subsp. irumaensis' AM-3603, which has been reported as a producer of irumamycin, ${ }^{2}$ was re-investigated. A new compound (1) with accurate mass $\left([\mathrm{M}+\mathrm{H}]^{+}, m / z\right.$ 1329.7126) and UV spectrum (absorption max at 228, 266, 276 and $285 \mathrm{~nm}$ ) was produced by the strain and the physicochemical properties did not correspond to known compounds in the existing database (Dictionary of Natural Products, CRC Press, FL, USA). Compound 1 was isolated from the culture broth and determined to be a new dimeric analog of oxazolomycin $\mathrm{A},{ }^{3}$ namely bisoxazolomycin (1). Furthermore, a hydrolyzed analog of oxazolomycin A, namely oxazolomycin A2 (2), along with oxazolomycin A (3) were also isolated from the culture broth. In this report, we describe the fermentation, isolation, structural elucidation and some biological activity of compounds 1,2 and 3.

\section{FERMENTATION AND ISOLATION}

The fermentation and isolation process is described in Supplementary Scheme S1. 'Streptomyces subflavus subsp. irumaensis' AM-3603, cultured on Waksman agar medium (1.0\% glucose, $0.5 \%$ peptone, $0.5 \%$ meat extract, $0.3 \%$ sodium chloride and $1.2 \%$ agar, $\mathrm{pH} 7.0$ ) was inoculated into a $500 \mathrm{ml}$-Erlenmeyer flask containing $100 \mathrm{ml}$ of YD medium (1.0\% glucose and $1.0 \%$ yeast extract, $\mathrm{pH}$ was not adjusted) and cultivated on a rotary shaker $\left(210\right.$ r.p.m.) at $27^{\circ} \mathrm{C}$ for 3 days. A 1 milliliter portion of the seed culture was inoculated into $500 \mathrm{ml}-$ Erlenmeyer flasks (total 58 flasks) each containing $100 \mathrm{ml}$ of $2.0 \%$ soluble starch, $0.5 \%$ glycerol, $1.0 \%$ defatted wheat germ, $0.3 \%$ meat extract, $0.3 \%$ dry yeast and $0.3 \% \mathrm{CaCO}_{3}$ (pH was not adjusted) and fermentation was carried out on a rotary shaker $\left(210\right.$ r.p.m.) at $27^{\circ} \mathrm{C}$ for 4 days.

The culture broth of strain AM-3603 was centrifuged at 3000 r.p.m. for $15 \mathrm{~min}$. The precipitate was extracted by ethanol and concentrated in vacuo. The extract was partitioned with ethyl acetate twice and concentrated to yield $836 \mathrm{mg}$. The ethyl acetate extract was applied to a silica gel FL100D column chromatography (32 i.d. $\times 40 \mathrm{~mm}$; Fuji Silysia Chemical, Aichi, Japan) and eluted with a stepwise gradient of $\mathrm{CHCl}_{3}-\mathrm{MeOH}(1: 0,100: 1,50: 1,9: 1,1: 1$ and 0:1 (v/v), each $100 \mathrm{ml})$. The elute fraction of $1: 1(52.6 \mathrm{mg})$ was purified by ODS column chromatography (15 i.d. $\times 20 \mathrm{~mm}$; Senshu Scientific, Tokyo, Japan), which was eluted with a stepwise gradient of acetonitrile $(10,20,30$, 40, 50, 60 and $100 \%(\mathrm{v} / \mathrm{v})$, each $20 \mathrm{ml})$. The fractions of 50 and $30 \%$ acetonitrile were dried in vacuo to yield $\mathbf{1}(8.7 \mathrm{mg})$ and $2(4.4 \mathrm{mg})$, respectively. Similarly, the 9:1 fraction $(244 \mathrm{mg})$ eluted from silica gel column chromatography was applied to an ODS column (20 i.d. $\times 50 \mathrm{~mm}$ ). The $30 \%$ acetonitrile fraction was dried in vacuo to yield 2 ( $7.4 \mathrm{mg})$. The $50 \%$ acetonitrile fraction $(58.5 \mathrm{mg})$ was purified by ODS column $(14$ i.d $\times 20 \mathrm{~mm})$ again, and then the $30-40 \%$ acetonitrile fraction was subsequently dried in vacuo to yield 3 (39.2 mg).

${ }^{1}$ Department of Genetics, Faculty of Science, Kasetsart University, Bangkok, Thailand; ${ }^{2}$ Kitasato Institute for Life Sciences, Kitasato University, Tokyo, Japan; ${ }^{3}$ Graduate School of Infection Control Sciences, Kitasato University, Tokyo, Japan and ${ }^{4}$ Center for Advanced Studies in Tropical Natural Resources, National Research-University-Kasetsart University (CASTNAR, NRU-KU), Kasetsart University, Bangkok, Thailand

${ }^{5}$ These authors contributed equally to this work.

Correspondence: Professor A Thamchaipenet, Department of Genetics, Faculty of Science, Kasetsart University, 50 Ngamwongwan Road, Ladyao, Chatuchak, Bangkok 10900, Thailand.

E-mail: arinthip.@ku.ac.th

or Professor T Nakashima, Kitasato Institute for Life Sciences, Kitasato University, 5-9-1 Shirokane, Minato-ku, Tokyo 108-8641, Japan.

E-mail: takuji@lisci.kitasato-u.ac.jp

Received 28 July 2017; revised 9 August 2017; accepted 24 August 2017; published online 27 September 2017 


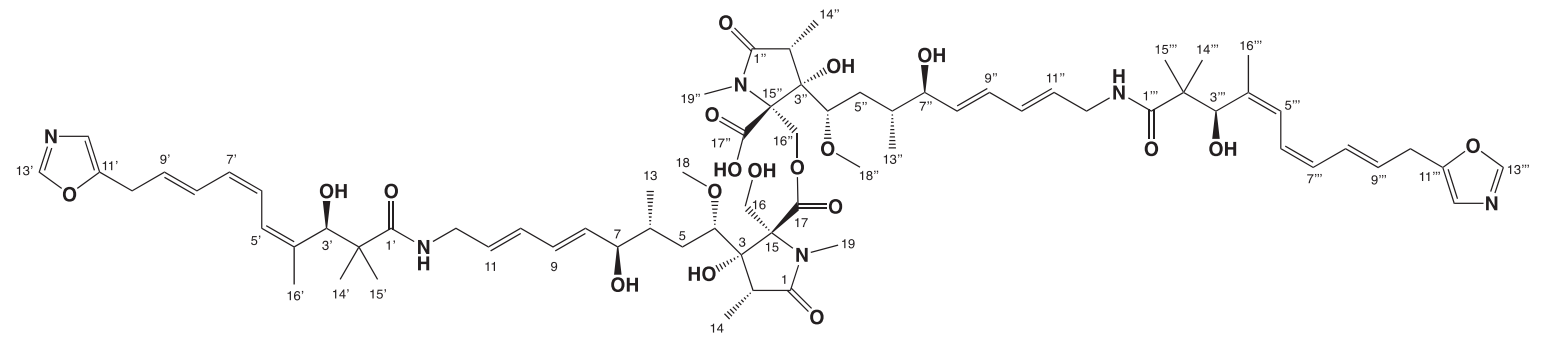

bisoxazolomycin (1)
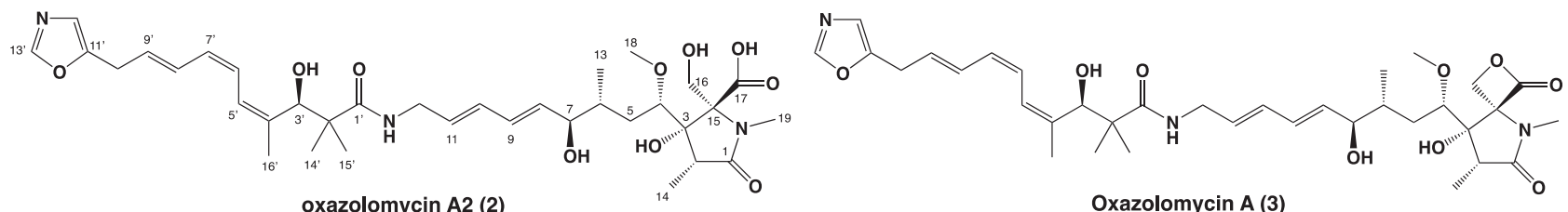

Figure 1 Structures of bisoxazolomycin (1), oxazolomycin A2 (2) and oxazolomycin (3)

\section{STRUCTURE ELUCIDATION}

Compound 3 was identified as oxazolomycin A by ${ }^{1} \mathrm{H}$ and ${ }^{13} \mathrm{C}$ NMR spectra (Supplementary Information), molecular formula, UV spectrum, IR spectrum and optical rotation (Supplementary Table S1), which were consistent with those described in the literatures. ${ }^{4,5}$ Oxazolomycin A (Figure 1) was discovered by Mori et al. ${ }^{3}$ and showed activity against P388 leukaemia cells.

Compound 2 was determined to have a molecular formula of $\mathrm{C}_{35} \mathrm{H}_{51} \mathrm{~N}_{3} \mathrm{O}_{10}$ by HR-ESI-MS $[\mathrm{M}+\mathrm{H}]^{+} \mathrm{m} / z 674.3667$ (consistent with a calculated value of 674.3647 for $\mathrm{C}_{35} \mathrm{H}_{52} \mathrm{~N}_{3} \mathrm{O}_{10}$ ), which was 18 mass units $\left(\mathrm{H}_{2} \mathrm{O}\right)$ larger than 3 . The UV spectrum and optical rotation of $\mathbf{2}$ were similar to those of $\mathbf{3}$, but the specific IR band of the $\beta$-lactone ring at $1825 \mathrm{~cm}^{-1}$ was not observed (Supplementary Table S1). The ${ }^{1} \mathrm{H}$ and ${ }^{13} \mathrm{C}$ NMR signals of 2 were assigned by combination of COSY, HSQC and HMBC spectra (Table 1 and Supplementary Figure S1). The NMR spectra were very similar to those of 3 but a difference in chemical shifts of the oxymethylene of $\mathrm{H}_{2}-16$ (3, 4.84 and 4.51 p.p.m.; 2, 4.22 and $4.16 \mathrm{ppm}$ ) was observed. These results suggest that $\mathbf{2}$ is a hydrolyzed analog of $\mathbf{3}$ at the $\beta$-lactone ring (Figure 1). Furthermore, 2 could be generated from hydrolysis of 3 with $0.1 \mathrm{M} \mathrm{NaOH}$ at room temperature (Supplementary Figure S2). Although 2 has been known as one of intermediates in the total synthesis of $3,{ }^{4}$ this is the first report of its isolation from among the metabolites of Streptomyces. Compound 2 was named as oxazolomycin A2.

Compound 1 was determined to have a molecular formula of $\mathrm{C}_{70} \mathrm{H}_{100} \mathrm{~N}_{6} \mathrm{O}_{19}$ by HR-ESI-MS $[\mathrm{M}+\mathrm{H}]^{+} \mathrm{m} / z 1329.7126$ (consistent with a calculated value of 1329.7116 for $\mathrm{C}_{70} \mathrm{H}_{101} \mathrm{~N}_{6} \mathrm{O}_{19}$ ). The UV spectra and optical rotation were similar to those of $\mathbf{2}$ and the specific IR band of $\beta$-lactone ring at $1825 \mathrm{~cm}^{-1}$ was not observed (Supplementary Table S1). The ${ }^{1} \mathrm{H}$ and ${ }^{13} \mathrm{C}$ NMR signals of $\mathbf{1}$ were assigned by combination of COSY, HSQC and HMBC spectra (Table 1 and Supplementary Figure S1). The ${ }^{1} \mathrm{H}$ and ${ }^{13} \mathrm{C}$ chemical shifts of oxazole-containing side chain from C-5 to C-13' and from $\mathrm{C}-5^{\prime \prime}$ to $\mathrm{C}-13^{\prime \prime \prime}$ were consistent with those of 2 . On the other hand, two sets of ${ }^{1} \mathrm{H}$ and ${ }^{13} \mathrm{C}$ signals were observed around $N$-methyl- $\gamma$-lactam segment. Therefore, $\mathbf{1}$ is an asymmetric dimer of 2 consisting $N$-methyl- $\gamma$-lactam segments I and II (Table 1 and Supplementary Figure S1). Segment I was confirmed by the ${ }^{1} \mathrm{H}_{-}{ }^{13} \mathrm{C}$ long-range correlations from $\mathrm{H}-2$ to $\mathrm{C}-1, \mathrm{C}-3$ and $\mathrm{C}-4$; from $\mathrm{H}_{3}-14-\mathrm{Me}$ to $\mathrm{C}-1$ and $\mathrm{C}-3$; from $\mathrm{H}_{2}-16$ to $\mathrm{C}-3, \mathrm{C}-15$ and $\mathrm{C}-17$; from $\mathrm{H}_{3}-18$-Me to $\mathrm{C}-4$; from $\mathrm{H}_{3}-19-\mathrm{Me}$ to $\mathrm{C}-1$ and $\mathrm{C}-15$ (Supplementary Figure S1). Segment II was also confirmed by the ${ }^{1} \mathrm{H}_{-}{ }^{13} \mathrm{C}$ long-range correlations from $\mathrm{H}-2^{\prime \prime}$ to $\mathrm{C}-1^{\prime \prime}, \mathrm{C}-3^{\prime \prime}$ and $\mathrm{C}-4^{\prime \prime}$; from $\mathrm{H}_{3}-14^{\prime \prime}-\mathrm{Me}$ to $\mathrm{C}-1^{\prime \prime}$ and $\mathrm{C}-3^{\prime \prime}$; from $\mathrm{H}_{2}-16^{\prime \prime}$ to $\mathrm{C}-3^{\prime \prime}$ and $\mathrm{C}-15^{\prime \prime}$; from $\mathrm{H}_{3}-18^{\prime \prime}-\mathrm{Me}$ to C- $4^{\prime \prime}$; from $\mathrm{H}_{3}-19^{\prime \prime}$-Me to C-1" and C-15" (Supplementary Figure S1). The chemical shifts of the oxymethylene of $\mathrm{H}_{2}-16$ (4.22 and 4.15 p.p. m.) in segment I were similar to those of 2 (4.22 and $4.16 \mathrm{ppm}$ ). However, the chemical shifts of the oxymethylene of $\mathrm{H}_{2}-16^{\prime \prime}$ (4.83 and $4.74 \mathrm{ppm}$ ) in segment II was observed downfield. Furthermore, 1 could be hydrolyzed to 2 in $0.1 \mathrm{M} \mathrm{NaOH}$ at room temperature (Supplementary Figure S2). Therefore, 1 was determined to be a new dimer of 2, which had an ester bond between C-17 and C-16" (Figure 1). Compound 1 was named as bisoxazolomycin.

\section{BIOLOGICAL ACTIVITY}

Cytotoxic activity of 1, 2 and 3 was measured by Cell Counting Kit-8 (Dojindo Laboratories, Kumamoto, Japan) in HL60 (Human promyelocytic leukemia cells). Briefly, HL60 $\left(3 \times 10^{5}\right.$ cells per well $)$ cell lines were seeded in 96-well plates and cultured in RPMI1640 (Nacalai Tesque, Kyoto, Japan) supplemented with $10 \%$ fetal bovine serum and $1 \%$ sodium pyruvate at $37^{\circ} \mathrm{C}$ under $5 \% \quad \mathrm{CO}_{2}$. After culturing for $1 \mathrm{~h}, \mathbf{1}, 2$ or 3 was dissolved in $\mathrm{MeOH}$ at appropriate concentrations was added into each well. After $48 \mathrm{~h}$ of incubation at $37^{\circ} \mathrm{C}$, WST- 8 solution was added to each well and incubated at $37^{\circ} \mathrm{C}$ for $4 \mathrm{~h}$. The absorbance at $460 \mathrm{~nm}$ of each well was measured using a Corona Grating Microplate Reader SH-9000 (Corona Electric, Ibaraki, Japan). Compound 1, 2 and $\mathbf{3}$ displayed cell growth inhibition against HL60 cells with $\mathrm{IC}_{50}$ values of $7 \mu \mathrm{M}, 20 \mu \mathrm{M}$ and $0.6 \mu \mathrm{M}$, respectively.

Compounds 1, 2 and 3 were investigated for antifungal activity, being tested against Candida albicans ATCC 64548, Mucor racemosus IFO 4581, Aspergillus niger ATCC 6275 and Saccharomyces cerevisiae ATCC 9763. Their antibacterial activity was examined against Kocuria rhizophila ATCC 9341, Bacillus subtilis ATCC 6633, Escherichia coli NIHJ, Xanthomonas campestis pv. oryzae KB 88, Pseudomonas aeruginosa IFO 3080, Staphylococcus aureus ATCC 6538p and Mycobacterium smegmatis ATCC 607 using the paper disc method ( $\phi 6 \mathrm{~mm}$, 
Table $1{ }^{1} \mathrm{H}$ and ${ }^{13} \mathrm{C}$ NMR spectral data of bisoxazolomycin (1) and oxazolomycin A2 (2)

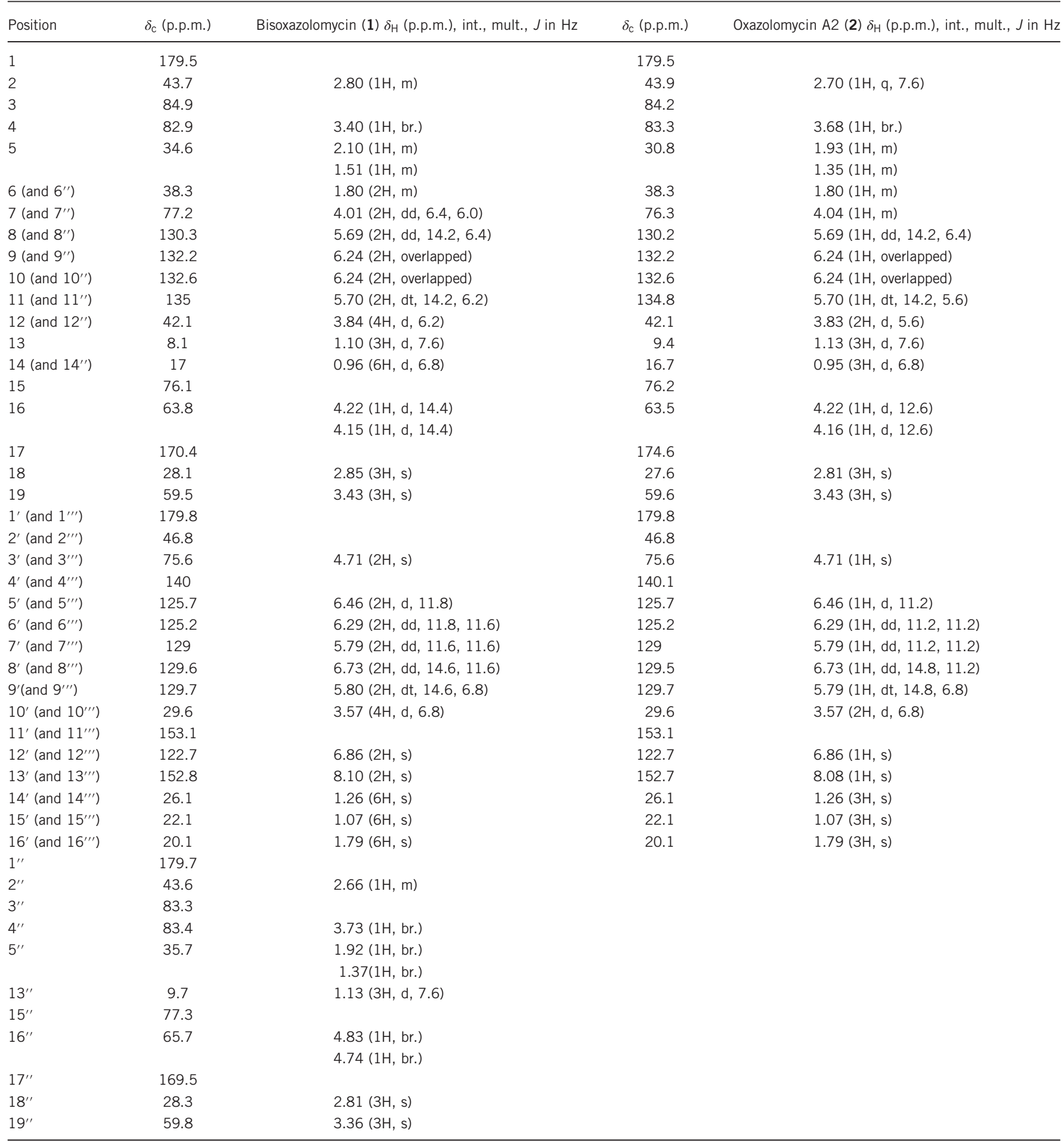

Advantec, Tokyo, Japan) at the concentrations of 30, 10, 3 and $1 \mu \mathrm{g}$ per disc. The compounds displayed no antifungal activity. Compound 3 showed antibacterial activity against K. rhizophila, B. subtilis, E. coli, $X$. campestis and S. aureus (Supplementary Table S2). Although 1 and 2 did exhibit antibacterial activity, it was less potent than 3 . It seems likely that the $\beta$-lactone ring of $\mathbf{3}$ plays an important role in the antibacterial activity.

\section{CONCLUSION}

We have discovered two new natural products, bisoxazolomycin (1) and oxazolomycin A2 (2), via PC screening technique. Both compounds showed cytotoxic and mildly antibacterial activities. PC screening is a powerful and comparatively simple technique to facilitate and expedite the discovery of new compounds from culture broths of microorganisms. 


\section{CONFLICT OF INTEREST}

The authors declare no conflict of interest.

\section{ACKNOWLEDGEMENTS}

We are grateful to Dr Masato Iwatsuki for helpful advice. This project was supported by JSPS and NRCT under the Japan - Thailand Research Cooperative Program, the Thailand Research Fund under Royal Golden Jubilee Ph.D. Program (Grant No. PHD/0205/2553) and the Institute for Fermentation, Osaka (IFO), Japan.
1 Nakashima, T., Takahashi, Y. \& Ōmura, S. Search for new compounds from Kitasato microbial library by physicochemical screening. Biochem. Pharmacol. 15, 42-55 (2017).

2 Ōmura, S. et al. Irumamycin, an antifungal 20-membered macrolide produced by a Streptomyces: Taxonomy, fermentation and biological properties. J. Antibiot. 37, 1572-1578 (1984).

3 Mori, T et al. Structure of oxazolomycin, a novel $\beta$-lactone antibiotic. Tetrahedron Lett. 26, 1073-1076 (1985).

4 Kawai, S., Kawabata, G., Kobayashi, A. \& Kawazu, K. Inhibitory effect of oxazolomycin on Crown Gall formation. Agric. Biol. Chem. 53, 1127-1133 (1989).

5 Eto, K., Yoshino, M., Takahashi, K., Ishihara, J. \& Hatakeyama, S. Total synthesis of Oxazolomycin A. Org. Lett. 13, 5398-5401 (2011).

Supplementary Information accompanies the paper on The Journal of Antibiotics website (http://www.nature.com/ja) 